\title{
Higher frequency of Central Pacific El Niño events in recent decades relative to past centuries
}

Mandy B. Freund ${ }^{1,2,3}$, Benjamin J. Henley ${ }^{1,2,4}$, David J. Karoly ${ }^{1,2,10}$, Helen V. McGregor ${ }^{5}$, Nerilie J. Abram ${ }^{6,7}$, and Dietmar Dommenget ${ }^{8,9}$

${ }^{1}$ School of Earth Sciences, University of Melbourne, Parkville, VIC 3010, Australia

${ }^{2}$ ARC Centre of Excellence for Climate System Science, University of Melbourne, Parkville, VIC 3010, Australia

${ }^{3}$ Australian-German Climate and Energy College, University of Melbourne, Parkville, VIC 3010, Australia

${ }^{4}$ ARC Centre of Excellence for Climate Extremes, University of Melbourne, Parkville, VIC 3010, Australia

${ }^{5}$ School of Earth, Atmospheric and Life Sciences, University of Wollongong, Wollongong, NSW 2522, Australia

${ }^{6}$ Research School of Earth Sciences, Australian National University, Canberra, ACT 2601, Australia

${ }^{7}$ ARC Centre of Excellence for Climate Extremes, Australian National University, Canberra, ACT 2601, Australia

${ }^{8}$ ARC Centre of Excellence for Climate Extremes, Monash University, Clayton, VIC 3800 , Australia

${ }^{9}$ School of Earth, Atmosphere and Environment, Monash University, Clayton, VIC 3800, Australia

${ }^{10}$ NESP Earth Systems and Climate Change Hub, CSIRO, Aspendale, VIC 3195, Australia

March 15, 2019 
El Niño events differ substantially in their spatial pattern and intensity. Canonical Eastern Pacific El Niño events have sea surface temperature anomalies that are strongest in the far eastern equatorial Pacific, whereas peak ocean warming occurs further west during Central Pacific El Niño events. The event types differ in their impacts on the location and intensity of temperature and precipitation anomalies globally. Evidence is emerging that Central Pacific El Niño events have become more common, a trend that is projected by some studies to continue with ongoing greenhouse warming. Here, we identify spatial and temporal patterns in observed sea surface temperatures that distinguish the evolution of Eastern and Central Pacific El Niño events in the tropical Pacific. We show that these patterns are recorded by a network of 27 seasonally resolved coral records, which we then use to reconstruct Central and Eastern Pacific El Niño activity for the past four centuries. We find a simultaneous increase in Central Pacific events and a decrease in Eastern Pacific events during the late 20th century that leads to a ratio of Central to Eastern Pacific events that is unusual in a multi-century context. Compared to the past four centuries, the most recent 30-year period includes fewer, but more intense Eastern Pacific El Niño events.

Canonical eastern Pacific (EP) El Niño events exhibit their largest sea surface temperature anomalies (SSTA) in the far eastern tropical Pacific near the Peruvian coast ${ }^{1}$. Over recent decades, peak warming during several El Niño events has been displaced approximately $11,000 \mathrm{~km}$, or $100^{\circ}$ longitude, westwards into the central equatorial Pacific. These El Niño events are described as Central Pacific (CP) El Niño, warm-pool El Niño ${ }^{2}$, El Niño Modoki ${ }^{3}$, or Dateline El Niño ${ }^{4}$. The displacement of maximum SSTA towards the central Pacific drives substantial shifts in atmospheric convection and circulation responses ${ }^{5-7}$, which alter the location and intensity of temperature and precipitation impacts associated with El Niño around the globe $3 ; 4 ; 8-11$.

Evidence is emerging that changes in El Niño Southern Oscillation (ENSO) behaviour have occurred during the instrumental period ${ }^{12-15}$. Following the climate regime shift in 1976/1977, zonal SSTA propagation during El Niño changed from westward to eastward ${ }^{16}$. Coincident with the shift to a positive phase of the Interdecadal Pacific Oscillation (IPO) ${ }^{16-18}$ in 1999/2000, Pacific trade winds have strengthened ${ }^{19 ; 20}$. Observations indicate increasing El Niño event amplitude ${ }^{21}$, strong decadal variations in event frequency ${ }^{22}$, changes in maximum SSTA propagation direction ${ }^{12 ; 23}$ and delays in the onset of El Niño events ${ }^{24}$.

Since the late 1990s there has been a higher number of CP events relative to EP events, unprecedented in instrumental records ${ }^{2 ; 21 ; 22}$. It is unclear whether this recent increase is part of natural climate variability ${ }^{25}$ or a consequence of anthropogenic climate change ${ }^{22}$. A precise picture of El Niño diversity is a challenge due to model deficiencies in simulating El Niño and the short and sparse coverage of instrumental observations across the equatorial Pacific ${ }^{25,26}$. In this study, we extend the record of El Niño diversity into the past using a network of coral data spanning the tropical Indo-Pacific ocean.

\section{Spatial and temporal patterns of El Niño types}

We use a network of 27 seasonally resolved coral records to reconstruct past EP and CP El Niño events (Methods and Supplementary Material). The network includes four Sr/Ca records (a proxy for SST) and 23 oxygen isotope $\left(\delta^{18} O\right)$ records. The $\delta^{18} O$ signal preserved in the coral banding is determined by the source isotopic composition 
of surrounding seawater $\delta^{18} O_{S W}$ and the equilibrium isotopic fractionation, inversely related to temperature between the seawater and carbonate ${ }^{27}$. The oxygen isotopes are fractionated throughout the annual cycle (thus preserve SST variations across the calendar year), and the $\delta^{18} O_{S W}$ is affected by advection of water masses with different isotope signatures, and precipitation-evaporation (P-E) changes. P-E changes also affect sea surface salinity (SSS), so $\delta^{18} O$ is often used as an SST, SSS or SST-SSS proxy. The relationship between salinity and $\delta^{18} O_{S W}$ is complex and can depend on local conditions ${ }^{28}$. In general, if sea surface salinity is relatively constant, $\delta^{18} O$ in corals is mainly determined by SST variability and vice versa. At the same time, highly variable SSS and SST variations can make the interpretation of $\delta^{18} O$ in corals more complex. We have carried out extensive testing of our methods and explored possible sources of error (Supplementary Material), including testing the $\delta^{18} O$ signal in the individual coral records in our network and our network as a whole (Supplementary Material 3.1.1). We find that all the coral $\delta^{18} O$ records in our network have a link to ENSO and all have high correlations to basin-wide SST and SSS, parameters which in turn vary with the spatial and temporal patterns of the different El Niño types.

Here we assess the shared tropical ocean and atmosphere climate signals across our coral network using Empirical Orthogonal Function (EOF) analysis and compare them with the spatial and temporal signals of EP and CP El Niño in instrumental SST. The observed spatial patterns of the EP and CP El Niño events are illustrated by EOFs of instrumental tropical Pacific SST anomalies (Fig.1a,b).

The well-known canonical EP pattern ${ }^{1}$ is represented by the first EOF and shows pronounced warming off the South American coast extending westwards. The instrumental EP SST pattern has a strong resemblance to the leading mode of the coral network (Fig.1c). The coral network captures the EP zonal dipole pattern, primarily because there is a strong negative contribution from coral sites in the western Pacific region.

CP El Niño, in contrast, appears as a zonal tripolar SST pattern in the instrumental data ${ }^{3}$ and is associated with the second EOF (Fig.1b). Warm SSTAs close to the dateline are flanked by cool SSTAs over the maritime continent and in the eastern equatorial Pacific. A tripole pattern also emerges from the second mode of the coral network (Fig.1d). The tripole pattern in the second EOF of instrumental and coral data has strong positive loadings from the western Pacific. This illustrates the ability of the coral network to capture the spatial signatures of CP as well as canonical EP El Niño.

The temporal evolution of the coral EOF modes is significantly correlated $(\mathrm{p}<0.001)$ with key ENSO indices (Fig. 1e,f) including the Niño Cold Tongue (NCT) and Niño Warm Pool (NWP) indices that discriminate between EP and CP El Niño, respectively ${ }^{17}$ (Methods). The first coral EOF is significantly correlated with the first SSTA EOF $(r=0.87)$, the NCT index $(r=0.68)$, the El Niño Modoki index (EMI, $r=0.46)$ and the Multivariate ENSO index (MEI, $r=0.87$ ). The coral network is also sensitive to temporal CP El Niño activity, as evidenced by the significant correlations between the second coral EOF and the second SSTA EOF $(r=0.52)$, as well as the NWP index $(r=0.35)$, Trans Niño index (TNI, $r=0.50)$ and El Niño Modoki index (EMI, $r=0.35$ ). This further shows that the first EOF of the coral network resembles a canonical EP El Niño signal, while the second EOF is associated with CP El Niño (Fig. 1e,f). 


\section{Seasonal distinction of El Niño events}

To differentiate between EP and CP types of past El Niño events we exploit the differences in the temporalspatial evolution in EP and CP El Niños. In the instrumental record the seasonal evolution of $\mathrm{CP}$ and EP events shows significant differences during the developing and peak phase (Fig.2a,b). During EP events, the Niño cold tongue region, expressed by the NCT index, shows stronger anomalies compared to CP events (Fig. 2a). In the warm pool, as expressed by the NWP index, SSTAs in CP events are higher in boreal summer and autumn (JJA-SON) and the largest differences between CP and EP events occurs in DJF. This seasonal distinction between EP and CP events is also recorded by the coral network (Fig. 2c,d). The second EOF mode of the coral data shows a similar seasonal evolution to instrumental temperatures in the warm pool. During the developing and peak phases, the coral network shows anomalously warm conditions during CP years compared to EP years. Based on this distinction between $\mathrm{CP}$ and $\mathrm{EP}$ events recorded by the coral network, we reconstruct the NCT index predominately by using the first coral EOF as a measure of canonical EP variability, and the NWP index for past CP variability.

\section{Seasonal reconstruction}

We first construct a continuous long-term history of seasonal El Niño variability in the central and eastern tropical Pacific for the last 400 years. We employ a nested regression approach, in which regressions are performed for each 'nest' of proxies which cover the same time period. We regress the NCT and NWP indices against principal components of the coral network (see Methods and Supplementary Material). The reconstructed indices represent $50 \%$ and $56 \%$ of the observed variance of NCT and NWP during the common period (1920-1984), respectively (Fig. 3). The skill of the reconstructions over the period 1617-2005 is indicated by positive values of the coefficient of efficiency (CE). The reconstructions are highly correlated with the instrumental indices and capture annual and seasonal variability (Fig. 3e,f, see insets). Tests of the sensitivity to different calibration periods, as expressed by the ensemble, produce only slight changes to the reconstructions (see Supplementary Fig. 34). The NCT reconstruction, related to EP events, shows best skill during the developing and peak season (JJA-DJF), with reduced skill during the decaying phase (MAM). This could be related to the well-known spring predictability barrier ${ }^{29}$. The NWP reconstruction captures the variability similarly well for all seasons. The two seasonal reconstructions of El Niño variability enable us, in conjunction with the differences in their seasonal evolution, to classify and distinguish El Niño events in the past.

\section{Four centuries of EP and CP El Niño events}

We identify EP and CP events by applying a decision classification tree (see Methods) to the reconstructed NCT and NWP indices (Fig. 3c-d). The decision tree was trained on instrumental data and independently identifies the seasonal evolution of EP and CP El Niño (Fig. 2). CP El Niño events occur frequently in past centuries ranging between 1-6 events within 30 year intervals (Fig.4a). We document 37 distinct CP El Niño events between 1617-1920 (Supplementary Table 7). EP events occur at a similar frequency to CP El Niño events and we identify a total of 27 EP events between 1617-1920 (Fig.4b). Although El Niño events are evident 
throughout the entire reconstruction, both types of El Niño events exhibit modulation in event frequency at the multidecadal timescale. However, the ratio of CP to EP events remained relatively stable in past centuries (Fig.4c). Our reconstructions show that with only a few brief exceptions (e.g. enhanced CP to EP activity in the late 1600s), EP events were as common as CP events prior to the 1980s. For both types of events, the intensity of maximum anomalies also remained relatively stable over the past four centuries, with generally lower amplitudes for CP than EP events.

In contrast, the reconstructed and observed sequence of El Niño event history for the 20th century shows a marked increase in CP El Niño event frequency. The number of CP events in the most recent period post-1980 has more than doubled compared to the pre-instrumental average, from 3.5 to 9 events per 30 years. At the same time, the number of EP events has remained low, at about 2 events per 30 years, which is within the frequency range of the last four centuries. Since the 1980s a higher proportion of El Niño events are of CP character, relative to EP character. The unprecedented increase of CP events and the simultaneous decrease of EP events during the late 20th century leads to a ratio of CP to EP El Niño events that is unusual during both the instrumental period and in the long-term context of past four centuries (Fig.4c).

Our result, of increased CP frequency, is robust and our additional tests demonstrate that the trend is not a function of chronological uncertainties, the declining availability of coral records back in time, methodological choices or low-frequency variability (see Supplementary Material). We note that the relationship between coral skeletal $\delta^{18} O$ and both SST and $\delta^{18} O_{S W}$ can vary between sites. Although there remains the potential for a small degree of signal damping or amplification by the combination of varying effects of $\operatorname{SST}$ and $\delta^{18} O_{S W}$ on coral $\delta^{18} O$, we find that the SST signal dominates at most sites and our analysis is robust to these site-specific sensitivities (see Supplementary material for detailed analysis).

Although the number of EP events has decreased since the 1980s, the intensity of EP events since this time has been high in the context of both the instrumental history and the reconstruction (Fig.4d). CP events appear not to have increased in intensity. Despite the potential limitations of coral-based reconstructions to capture precise maximum amplitudes related to SSTA in Niño3 and Niño4 during El Niño events, the observed intensity of recent EP events is stronger than in the full length of the instrumental (post 1920) and reconstructed records. We note that the coral reconstruction underestimates the intensity of the 1982/1983 and 1997/1998 El Niño events, but does capture the intensity of other El Niño events identified in the instrumental record (see Supplementary Material 5.2.5 for discussion). Notwithstanding this caveat, our reconstructions offer a long-term context for seasonal El Niño variability that demonstrates the unusual recent increase in CP frequency and EP amplitude. Assuming that the reconstructions are representative of past amplitudes, the three most recent EP events $(1982,1997,2015)$ are of unprecedented amplitude relative to the last four centuries, and have seasonal instrumental SSTA in the tropical Pacific exceeding $2.4^{\circ} \mathrm{C}$.

The diversity of El Niño events and the recent changes in their relative frequency and intensity challenge efforts to provide seasonal to decadal predictions ${ }^{30,31}$. In $2014 / 2015$ the 'failed' El Niño ${ }^{32,33}$ is categorised by our classification scheme as a CP event, while the following year 2015/2016 closely resembled a canonical EP pattern and resulted in one of the strongest events in the past 400 years. This evolution of events appears unusual in the instrumental record, however our reconstructions provide evidence that this sequence occurred 
at least 8 times in the past 400 years (Fig.4c,).

Our 400 year reconstruction of EP and CP El Niño events gives a valuable long-term context to observational studies by showing that the shift towards more frequent $\mathrm{CP}$ events in recent decades ${ }^{3,34,2}$ and a tendency towards more extreme EP events ${ }^{21}$ is unusual in the past four centuries. During the last three decades, CP events have occurred more than four times as often as EP events (Fig. 4c, Supplementary Fig.S34). Under a high emissions scenario a projected increase in the $\mathrm{CP} / \mathrm{EP}$ event ratio to 5:1 could occur by the end of the century ${ }^{35,22}$. Our reconstructions may provide evidence for an earlier or more extreme shift towards CPtype El Niño events than is projected from climate model simulations. Alternatively, this difference could be related to the difficulties representing the full range of ENSO diversity and variability in climate models ${ }^{36}$. Large uncertainties in the tropical response to climate change may arise from a poorly constrained mean state, manifested by a pronounced Pacific cold tongue bias ${ }^{37}$, double Intertropical Convergence Zone ${ }^{38}$ and diverging dynamical responses ${ }^{39}$. This presents challenges for future projections of CP-type El Niño events, and their emerging global impacts. Expansion of the seasonally resolved coral network to include more paired Sr/Ca and $\delta^{18} O$ records would provide greater certainty around the site-specific sensitivities of $\delta^{18} O$ to $\mathrm{SST}$ and $\delta^{18} O_{S W}$ and more precise reconstructions of past SST and ENSO events. However, our new reconstructions of El Niño diversity provide a valuable baseline for constraining past and recent changes in El Niño diversity and improving our understanding of changing El Niño behaviour in a warming world. 


\section{Methods}

For a detailed description and schematic of the methods see Supplementary Material.

\section{Instrumental El Niño indices}

We use the Niño Warm Pool (NWP) index and the Niño Cold Tongue index (NCT) ${ }^{17}$ as instrumental targets for our reconstruction (see Supplementary methods section 2.1 for details). The NWP and NCT indices are a linear combination of monthly area average SSTA from the Niño3 $\left(5^{\circ} \mathrm{N}-5^{\circ} \mathrm{S}, 150^{\circ}-90^{\circ} \mathrm{W}\right)$ and Niño4 $\left(5^{\circ} \mathrm{N}-\right.$ $\left.5^{\circ} \mathrm{S}, 160^{\circ} \mathrm{E}-150^{\circ} \mathrm{W}\right)$ regions and are computed from the Hadley Centre Sea Ice and Sea Surface Temperature (HadISST) dataset ${ }^{40}$ from 1870-2015 (http://www.metoffice.gov.uk/hadobs/hadisst/). Due to the scarcity of maritime observations in the early period, all analysis using the HadISST dataset is limited to the period after 1920. Differences between instrumental datasets, data quality and the implications for the reconstruction are discussed in Supplementary Material section 2.1.

\section{Data aggregation and pre-processing}

We compiled a network of 27 high-resolution coral-derived records, 23 based on stable oxygen isotope ratios $\left(\delta^{18} O\right)$ and 4 on $\mathrm{Sr} / \mathrm{Ca}$ ratios (Supplementary Fig. 11, Supplementary Table 3$)$. The records are either directly geochemically linked to changes in SST or related to coupled ocean-atmosphere system (SST, and seawater $\delta^{18} O$, where seawater $\delta^{18} O$ can be modulated by precipitation, evaporation and advection). The network is composed of records that are: (i) located in the tropical Indo-Pacific ocean, equatorward of $20^{\circ} \mathrm{N} / \mathrm{S}$, and from $90^{\circ} \mathrm{E}-290^{\circ} \mathrm{E}$ $\left(70^{\circ} \mathrm{W}\right)$, (ii) have sub-annual temporal resolution of least 4 measurements per year, and (iii) start prior to 1901. The temporal resolution of the coral records varies from sub-annual to monthly (see Supplementary Tables $2 \& 3$ for details of the coral records). The majority of the records were previously used to reconstruct ENSO or tropical variability at an annual timescale ${ }^{41,42,43}$. Pre-processing of coral records is required due to their non-gaussian distribution, unevenly sampled time intervals, different temporal resolutions and a pronounced seasonal cycle. We first apply a transformation to obtain gaussian-distributed data ${ }^{44}$. We subsample the records onto a regular time grid of 4 samples per year: MAM (March - May), JJA (June - August), SON (September - November), DJF (December - February) using a weighted averaging procedure (see Supplementary Methods section 3.2). We remove multi-centennial trends using a 150-year spline ${ }^{42}$. The resulting seasonal records contain a small number of missing values $(<1 \%)$ which we impute by the regularised expectation maximisation (RegEM) method $^{45}$. All records are normalised to have a zero mean and unit standard deviation. Pre-processing steps and methodological choices are discussed in Supplementary section 3.3.

\section{Reconstruction}

The seasonally resolved coral records from the tropical Pacific are used to reconstruct seasonal means (MAM, JJA, SON, DJF) of the Niño Warm Pool (NWP) and Niño Cold Tongue (NCT) indices since 1600. We employ a nested, principal component regression (PCR) approach using variational Bayesian inference ${ }^{46 ; 47}$. Principle component analysis is applied to each nest (subset) of proxy records which cover the same time 
period. The principal components are then considered predictors in a multiple regression model in which the regression coefficients are estimated by variational inference (details in Supplementary section 5.2.1). This method enables us to derive estimated regression coefficients (traditionally obtained by maximum likelihood fitting Supplementary Fig. 28) using automatic relevance determination which shrinks irrelevant coefficients during inference ${ }^{48 ; 49}$. The normalised coral records are decomposed into their leading Empirical Orthogonal function (EOFs) by principal component analysis. We retain only the first $\boldsymbol{n}$ principal components that account for $80 \%$ of the total variance as possible predictors to avoid possible co-linearity effects and remove non-climate related noise. Multiple linear regression is performed to reconstruct continuous NWP and NCT indices by regressing the instrumental target during the calibration period against the retained principal components of the coral network. Each reconstruction is evaluated by skill statistics including the coefficient of determination $\left(R^{2}\right)$, the reduction of error (RE) and the coefficient of efficiency $(\mathrm{CE})^{50}$ (details in Supplementary section $5.2 .2)$.

We use a nested approach to maximise the length of our reconstruction. Based on the availability of the records (Supplementary Table S6), we build sequential subsets (nests) of records in which each of the subset reconstructions is adjusted to have a common mean and standard deviation with the most replicated nest. Continuous reconstructions are created by splicing reconstructions with maximal time integrated RE.

The reconstruction is tested using different calibration and verification periods (see also Supplementary Fig. 34). In determining our calibration interval we consider the longest possible calibration and verification periods, the increasing quality of SST datasets with time (Supplementary Fig.2), ENSO regime shifts in the mid-to-late $1970 \mathrm{~s}^{16}$ and the different phases of multidecadal variability. To encompass these variables we select a common overlapping period of 1920-1984 as our calibration/validation domain. A block-wise validation scheme is applied, with alternating calibration and verification blocks of at least 10 years (except for the latest) covering $2 / 3$ and $1 / 3$ of the years respectively, resulting in an alternating sequence of 35 blocks. An ensemble of reconstructions is built with different calibration and verification periods. The reconstruction with the highest time-integrated RE is used as the final reconstruction. This reconstruction uses a calibration period of 1920-1939, 1960-1969, 19801984 and a verification period of 1940-1959/1970-1979. Reconstruction uncertainty is quantified by simulating the errors using an autoregressive process fitted to the residuals in the verification period (see Supplementary Fig 29). The estimate of the confidence level is obtained with a 1000 realisations bootstrap approach by adding red noise to the records. The sensitivity of the reconstruction to different calibration/verification periods, individual records and EOFs retained is described in the Supplementary Material.

\section{Classification}

We perform objective supervised machine learning classification to identify the two types of El Niño events. Classification decision and regression tree analysis (CART) partitions data along the predictor axes and enables categorical prediction based on the input variables ${ }^{51}$. The decision tree (Supplementary Fig. S32a) is built to classify three categorical classes: EP type, CP type or no El Niño event. Each branch indicates a binary decision and is conditioned by previous decisions. The decision tree is used as a predictive model to infer classification rules and is based on the seasonal evolution of El Niño events. We identify the seasonal trajectory that leads to 
the two types of El Niño events. This implies that those events are phase-locked to a particular season during the developing stage and mature phase and locked to relative intensities.

The seasonal (MAM, JJA, SON, DJF) time series of the two Niño indices (NWP and NCT) result in 8 predictor variables. Although this non-parametric analysis is not subject to linear constraints, its predictive accuracy is sensitive to changes in the data, and it assumes temporal stationarity. Therefore, prior to the analysis all predictor variables are adjusted individually to have a constant mean and stable moving variance (i.e. homoscedasticity) by normalising the NWP and NCT indices within a moving window of 30 years length. This is comparable to a high-pass filter normalisation.

We train a classification tree based on the normalised instrumental NWP and NCT during the period 19502005. The decision tree categorises two types of El Niño based on the seasonal means of NWP and NCT. During the period 1950-2005 EP El Niño events include the years: 1951, 1957, 1965, 1972, 1976, 1982, 1986,1997 while CP El Niño years are identified as: 1963, 1968, 1977, 1979, 1987, 1991, 1994, 2002, 2004 ${ }^{52}$, excluding weak events (see composite plots for details, Supplementary Fig.S5). The majority of EP and CP years coincide with years previously identified despite differing methodological approaches and subjective thresholds (see Supplementary Material section 2.3). Next, we apply this decision tree to our adjusted reconstructed Niño indices during the reconstruction period (1617-2005) and to the most recent instrumental data for the years from 1920 to 2017.

The performance of the classifiers is evaluated for the instrumental and reconstruction base period (19502005) by confusion matrix, also known as a contingency table. The tree performs well on the basis of the metrics for accuracy, precision, sensitivity, F-score and Matthews correlation coefficient (MCC) (Supplementary Methods \& Table S7). The classification tree is evaluated by various cross-validation techniques applied to the instrumental data. It achieves an accuracy of $97 \%$, precision of $88 \%$ and a false detection rate of $3 \%$ (Supplementary Table S8). For the reconstructed record, the accuracy is reduced to 87\%, precision of $80 \%$ and false detection rate of $10 \%$. The confusion matrices indicate high fidelity in distinguishing between an El Niño event and no event and have an especially high ability to differentiate between the two types of El Niño events.

Based on the decision classification tree, we obtain an estimate of past El Niño events, their frequency, character (type) and intensity. The intensity is derived from the back transform of the NCT and NWP indices into Niño 3 and Niño 4 anomalies. The intensity is then based on the maximum SSTA in the Niño 3 and Niño 4 region. 


\section{References}

${ }^{1}$ Rasmusson, E. M. \& Carpenter, T. H. Variation in tropical sea surface temperature and surface wind fields associated with the Southern Oscillation/El Niño. Monthly Weather Review 110, 354 (1982).

${ }^{2}$ Kug, J.-S., Jin, F.-F. \& An, S.-I. Two Types of El Niño Events: Cold Tongue El El Niño and Warm Pool El El Niño. Journal of Climate 22, 1499-1515 (2009).

${ }^{3}$ Ashok, K., Behera, S. K., Rao, S. A., Weng, H. \& Yamagata, T. El Niño Modoki and its possible teleconnection. Journal of Geophysical Research 112, C11007 (2007).

${ }^{4}$ Larkin, N. K. \& Harrison, D. E. Global seasonal temperature and precipitation anomalies during El Niño autumn and winter. Geophysical Research Letters 32, L16705 (2005).

${ }^{5}$ Di Lorenzo, E. et al. Central Pacific El Niño and decadal climate change in the North Pacific Ocean. Nature Geoscience 3, 762-765 (2010).

${ }^{6}$ Yu, J.-Y., Kao, H.-Y., Lee, T. \& Kim, S. T. Subsurface ocean temperature indices for Central-Pacific and Eastern-Pacific types of El Niño and La Niña events. Theoretical and Applied Climatology 103, 337-344 (2010).

${ }^{7}$ Graf, H.-F. \& Zanchettin, D. Central Pacific El Niño, the "subtropical bridge," and Eurasian climate. Journal of Geophysical Research 117, D01102-10 (2012).

${ }^{8}$ Wang, G. \& Hendon, H. H. Sensitivity of Australian Rainfall to Inter-El Niño Variations. Journal of Climate 20, 4211-4226 (2007).

${ }^{9}$ Taschetto, A. S. \& England, M. H. El Niño Modoki Impacts on Australian Rainfall. Journal of Climate 22, $3167-3174$ (2009).

${ }^{10}$ Cai, W. \& Cowan, T. La Niña Modoki impacts Australia autumn rainfall variability. Geophysical Research Letters 36, L12805-4 (2009).

${ }^{11}$ Frauen, C., Dommenget, D., Tyrrell, N., Rezny, M. \& Wales, S. Analysis of the Nonlinearity of El NinoSouthern Oscillation Teleconnections. Journal of Climate 27, 6225-6244 (2014).

${ }^{12}$ Fedorov, A. V. \& Philander, S. G. Is El Niño changing? Science 288, 1997-2001 (2000).

${ }^{13}$ Ren, H.-L., Jin, F.-F., F Stuecker, M. \& Xie, R. ENSO Regime Change since the Late 1970s as Manifested by Two Types of ENSO. Journal of the Meteorological Society of Japan. Ser. II 91, 835-842 (2013).

${ }^{14} \mathrm{An}, \mathrm{S}$. I. \& Wang, B. Interdecadal change of the structure of the ENSO mode and its impact on the ENSO frequency. Journal of Climate 13, 2044-2055 (2000).

${ }^{15}$ Aiken, C. M., Santoso, A., McGregor, S. \& England, M. H. Optimal forcing of ENSO either side of the 1970's climate shift and its implications for predictability. Climate Dynamics 45, 1-19 (2015).

${ }^{16}$ Trenberth, K. E. \& Stepaniak, D. P. Indices of El Niño evolution. Journal of Climate 14, 1697-1701 (2001). 
${ }^{17}$ Ren, H.-L. \& Jin, F.-F. Niño indices for two types of ENSO. Geophysical Research Letters 38, L04704 (2011).

${ }^{18}$ Henley, B. J. et al. A Tripole Index for the Interdecadal Pacific Oscillation. Climate Dynamics 45, 1-14 (2015).

${ }^{19}$ L'Heureux, M. L. Recent multidecadal strengthening of the Walker circulation across the tropical Pacific. Nature Climate Change 3, 571-576 (2013).

${ }^{20}$ England, M. H. et al. Recent intensification of wind-driven circulation in the Pacific and the ongoing warming hiatus. Nature Climate Change 4, 222-227 (2014).

${ }^{21}$ Lee, T. \& McPhaden, M. J. Increasing intensity of El Niño in the central-equatorial Pacific. Geophysical Research Letters 37, L14603 (2010).

${ }^{22}$ Yeh, S.-W. et al. El Niño in a changing climate. Nature 461, 511-514 (2009).

${ }^{23}$ McPhaden, M. J. \& Zhang, X. Asymmetry in zonal phase propagation of ENSO sea surface temperature anomalies. Geophysical Research Letters 36, L13703-6 (2009).

${ }^{24}$ Wang, B. Interdecadal changes in El Nino onset in the last four decades. Journal of Climate 8, 267-285 (1995).

${ }^{25}$ Newman, M., Shin, S.-I. \& Alexander, M. A. Natural variation in ENSO flavors. Geophysical Research Letters 38, L14705 (2011).

${ }^{26}$ Capotondi, A. et al. Understanding ENSO Diversity. Bulletin of the American Meteorological Society 96, $921-938(2015)$.

${ }^{27}$ Weber, J. N. \& Woodhead, P. M. Temperature Dependence of Oxygen-18 Concentration in Reef Coral Carbonates. Journal of Geophysical Research 77, 463-\& (1972).

${ }^{28}$ Grottoli, A. G. \& Eakin, C. M. A review of modern coral $\delta 18 \mathrm{O}$ and $\Delta 14 \mathrm{C}$ proxy records. Earth-Science Reviews 81, 67-91 (2007).

29 Torrence, C. \& Webster, P. J. The annual cycle of persistence in the El Nino Southern Oscillation. Quarterly Journal of the Royal Meteorological Society 124, 1985-2004 (1998).

${ }^{30}$ Liu, Y. et al. Recent enhancement of central Pacific El Niño variability relative to last eight centuries. Nature Communications 8, 15386 (2017).

${ }^{31}$ McPhaden, M. J. Playing hide and seek with El Niño. Nature Climate Change 5, 791-795 (2015).

${ }^{32}$ Menkes, C. E. et al. About the role of Westerly Wind Events in the possible development of an El Nino in 2014. Geophysical Research Letters 41, 6476-6483 (2014).

${ }^{33}$ Chen, D. et al. Strong influence of westerly wind bursts on El Niño diversity. Nature Geoscience 8, 1-8 (2015). 
${ }^{34}$ Kao, H.-Y. \& Yu, J.-Y. Contrasting Eastern-Pacific and Central-Pacific Types of ENSO. Journal of Climate 22, 615-632 (2009).

${ }^{35}$ Kim, S. T. \& Yu, J.-Y. The two types of ENSO in CMIP5 models. Geophysical Research Letters 39, L11704 (2012).

${ }^{36}$ Yu, Y., Dommenget, D., Frauen, C., Wang, G. \& Wales, S. ENSO dynamics and diversity resulting from the recharge oscillator interacting with the slab ocean. Climate Dynamics 46, 1665-1682 (2015).

${ }^{37}$ Liu, Z. Y., Vavrus, S., He, F., Wen, N. \& Zhong, Y. F. Rethinking tropical ocean response to global warming: The enhanced equatorial warming. Journal of Climate 18, 4684-4700 (2005).

${ }^{38}$ Latif, M. et al. ENSIP: the El Niño simulation intercomparison project. Climate Dynamics 18, 255-276 (2001).

${ }^{39}$ Chen, C., Cane, M. A., Wittenberg, A. T. \& Chen, D. ENSO in the CMIP5 Simulations: Life Cycles, Diversity, and Responses to Climate Change. Journal of Climate 30, 775-801 (2017).

${ }^{40}$ Rayner, N. A., Parker, D. E. \& Horton, E. B. Global analyses of sea surface temperature, sea ice, and night marine air temperature since the late nineteenth century. Journal of Geophysical Research 108, 4407 (2003).

${ }^{41}$ Tierney, J. E. et al. Tropical sea surface temperatures for the past four centuries reconstructed from coral archives. Paleoceanography 30, 226-252 (2015).

${ }^{42}$ Emile-Geay, J., Cobb, K. M., Mann, M. E. \& Wittenberg, A. T. Estimating Central Equatorial Pacific SST Variability over the Past Millennium. Part II: Reconstructions and Implications. Journal of Climate 26, 2329-2352 (2013).

${ }^{43} \mathrm{Li}$, J. et al. Interdecadal modulation of El Niño amplitude during the past millennium. Nature Climate Change 1, 114-118 (2011).

${ }^{44}$ Emile-Geay, J. \& Tingley, M. P. Inferring climate variability from nonlinear proxies: application to paleoENSO studies. Climate of the Past Discussions 11, 2763-2809 (2015).

${ }^{45}$ Schneider, T. Analysis of incomplete climate data: Estimation of mean values and covariance matrices and imputation of missing values. Journal of Climate 14, 853-871 (2001).

${ }^{46}$ Mathys, C. A Bayesian foundation for individual learning under uncertainty. Frontiers in Human Neuroscience 5, 39 (2011).

${ }^{47}$ Bishop, C. Pattern Recognition and Machine Learning (Information Science and Statistics), 1 st edn. 2006. corr. 2nd printing edn (Springer, 2007).

${ }^{48}$ Daunizeau, J., Friston, K. J. \& Kiebel, S. J. Variational Bayesian identification and prediction of stochastic nonlinear dynamic causal models. Physica D 238, 2089-2118 (2009).

${ }^{49}$ Blei, D. M., Kucukelbir, A. \& McAuliffe, J. D. Variational Inference: A Review for Statisticians. Journal of the American Statistical Association 112, 859-877 (2017). 
${ }^{50}$ Cook, E. R., Meko, D. M., Stahle, D. W. \& Cleaveland, M. K. Drought reconstructions for the continental United States*. Journal of Climate 12, 1145-1162 (1999).

${ }^{51}$ Krzywinski, M. \& Altman, N. Classification and regression trees. Nature Methods 14, 755-756 (2017).

${ }^{52}$ Yeh, S.-W., Wang, X., Wang, C. \& Dewitte, B. On the Relationship between the North Pacific Climate Variability and the Central Pacific El Niño. Journal of Climate 28, 663-677 (2015). 


\section{Acknowledgements}

M.F., D.K., and D.D. are supported by the Australian Research Council (ARC) Centre of Excellence for Climate System Science (CE110001028). B.J.H. is supported through an ARC Linkage Project (LP150100062) and is an Associated Investigator of the ARC Centre of Excellence for Climate Extremes. H.V.M. acknowledges support from ARC Future Fellowship (FT140100286). N.J.A. is supported by the ARC Centre of Excellence for Climate Extremes (CE170100023) and a Future Fellowship (FT160100029). D.K. is currently supported by the Earth Systems and Climate Change Hub in the Australian Government's National Environmental Science Program.

\section{Author Contributions}

M.F. conceived and designed the study, with input from B.H. and D.K. M.F. led the development of methods, the analysis and the writing of the manuscript. Expert contributions and oversight came from B.H., D.K., H.M., N.A. and D.D. All authors contributed to discussions that shaped the study and the manuscript.

\section{Financial Interests statement}

The authors declare no competing financial interests.

\section{Corresponding author}

Mandy B. Freund, Mandy.Freund@csiro.au

\section{Data availability}

The proxy records with persistent identifier (doi/URL) are listed in Supplementary Table S4. Primary input and output data are archived by the National Oceanic and Atmospheric Administration (NOAA) for routine access and use (https://www.ncdc.noaa.gov/paleo/study/26270).

\section{Code availability}

The code associated with this paper is available on request from M.F. 


\section{List of Figures}

1 El Niño diversity in instrumental and coral data a,b First and second EOF-mode of seasonal instrumental SSTA (HadISST) in the tropical Pacific $\left(140^{\circ} \mathrm{E}-80^{\circ} \mathrm{W}, 30^{\circ} \mathrm{S}-30^{\circ} \mathrm{N}\right)$ regressed onto boreal summer (JJA) SSTA. c,d Coral network EOF-modes regressed onto SSTA during JJA and coral site loadings. e,f Comparison of instrumental SSTA-derived EOF modes (coloured bars) and coral (black line) EOF modes (normalised time series) with various ENSO indices (Niño Cold Tongue index (NCT), Niño Warm Pool index (NWP), El Niño Modoki index (EMI), Multivariate ENSO index (MEI) and Trans Niño index (TNI)). Significant correlations (r) between coral network EOF modes and individual indices are shown. All results based on common period 1950-1984. The EOF time series are normalised to facilitate visual comparison as the amipltude is essentially arbitrary.

2 Seasonal distinction of EP and CP El Niño events. Instrumental composite of Niño Cold Tongue index (NCT) (a) and Niño Warm Pool index (NWP) (b) for EP and CP events (19502005) during different seasons. Coral network composite of the first (c) and second (d) EOF mode during EP and CP events (1950-1984). Average (line), $25^{\text {th }}-75^{\text {th }}$ percentile range (dark shading) and full range (light shading). Grey shading indicates seasons that are statistically different using a t-test (Instrumental:p $<0.05$, Coral network:p $<0.1) \ldots \ldots \ldots \ldots \ldots$

3 Reconstruction of seasonal El Niño indices. a, Comparison between the reconstructed (black line) and instrumental (orange line) Niño Cold tongue index (NCT) and b, Niño Warm pool index (NWP) during instrumental period $\mathbf{c}, \mathbf{d}$, NCT and NWP seasonal reconstructions (dark lines), ensemble spread (light shading), 30-year low-pass filtered (blue lines) reconstructions and number of records. e,f, Verification statistics. Coefficient of Efficiency (CE) and explained variance $\left(R^{2}\right)$ for most replicated (sel, dark lines) and ensemble spread (ens, shaded envelopes). Insets show the median (red line), most replicated (red dot), interquartile range (grey boxes) and full range (whiskers) of ensemble spread of $\mathrm{CE}$ within seasons. . . . . . . . . . . . . .

4 El Niño event diversity over the past four centuries. a,b,c, Number of CP El Niño (a), EP El Niño events (b) and ratio of CP to EP events (c) in sliding 30-yr windows; instrumental (red), most-replicated reconstruction (teal), bootstrapped median (black) and uncertainty range in grey shadings $\left(75^{\text {th }}, 90^{\text {th }}, 95^{\text {th }}\right.$ percentiles) shown. Event occurrence frequencies (per decade, insets) in 50-yr periods; individual events indicated by markers at zero line. Vertical red dotted line indicates most recent window, centred on 2001. d, Intensity of CP and EP El Niño events obtained by the NWP/NCT indices; instrumental events outlined in black; error bars indicate $95 \%$ confidence interval from bootstrapping. Small triangles show the number of contributing records when data availability is limited. 


\section{Main Figures}
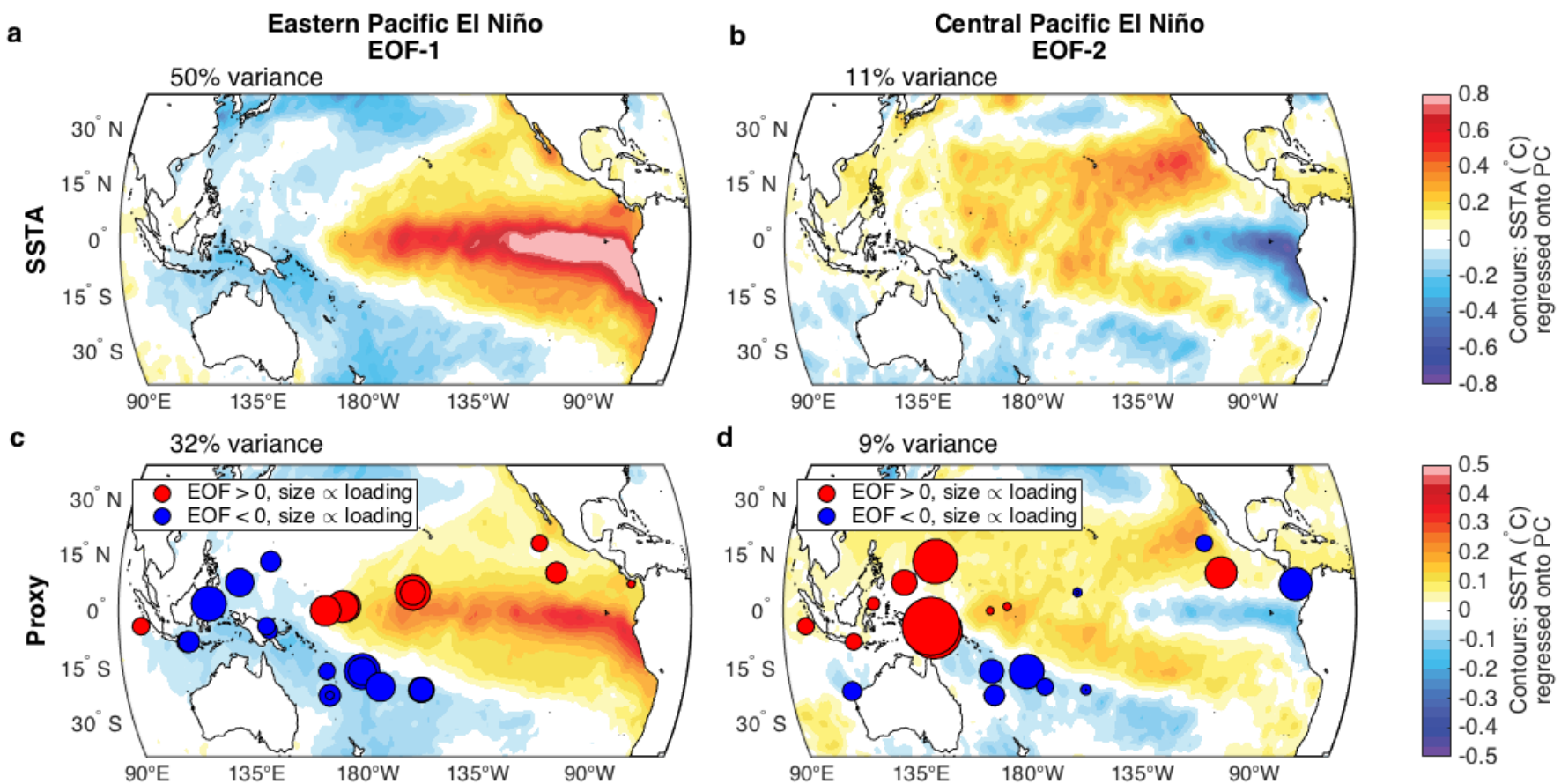

d

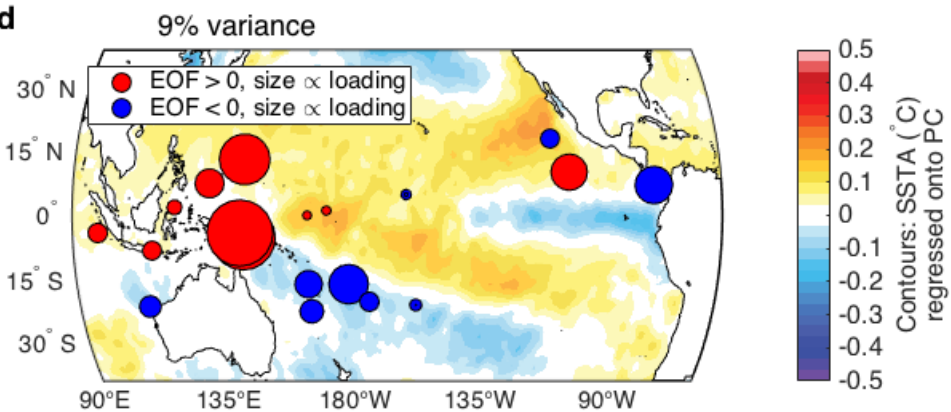

f
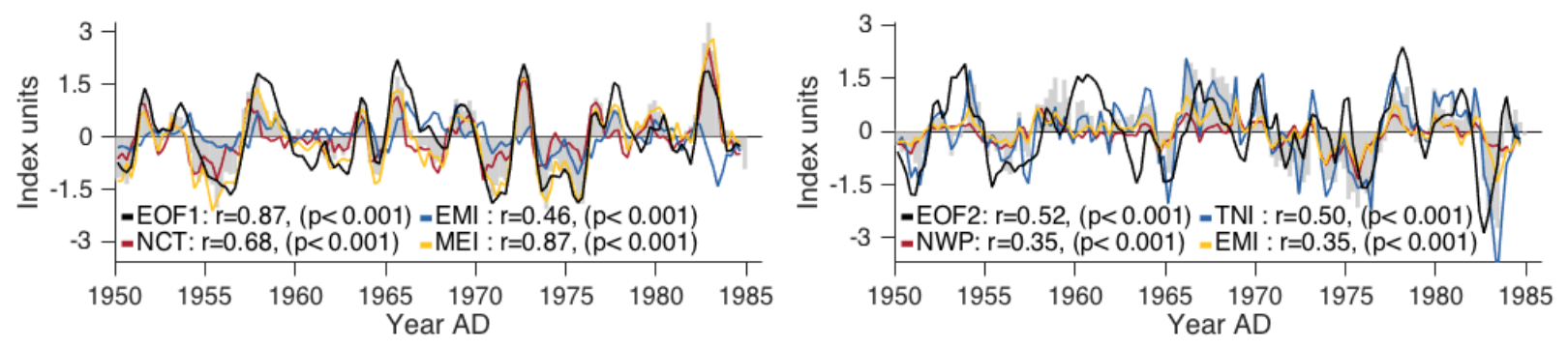

Figure 1: El Niño diversity in instrumental and coral data a,b First and second EOF-mode of seasonal instrumental SSTA (HadISST) in the tropical Pacific $\left(140^{\circ} \mathrm{E}-80^{\circ} \mathrm{W}, 30^{\circ} \mathrm{S}-30^{\circ} \mathrm{N}\right)$ regressed onto boreal summer (JJA) SSTA. c,d Coral network EOF-modes regressed onto SSTA during JJA and coral site loadings. e,f Comparison of instrumental SSTA-derived EOF modes (coloured bars) and coral (black line) EOF modes (normalised time series) with various ENSO indices (Niño Cold Tongue index (NCT), Niño Warm Pool index (NWP), El Niño Modoki index (EMI), Multivariate ENSO index (MEI) and Trans Niño index (TNI)). Significant correlations (r) between coral network EOF modes and individual indices are shown. All results based on common period 1950-1984. The EOF time series are normalised to facilitate visual comparison as the amipltude is essentially arbitrary. 


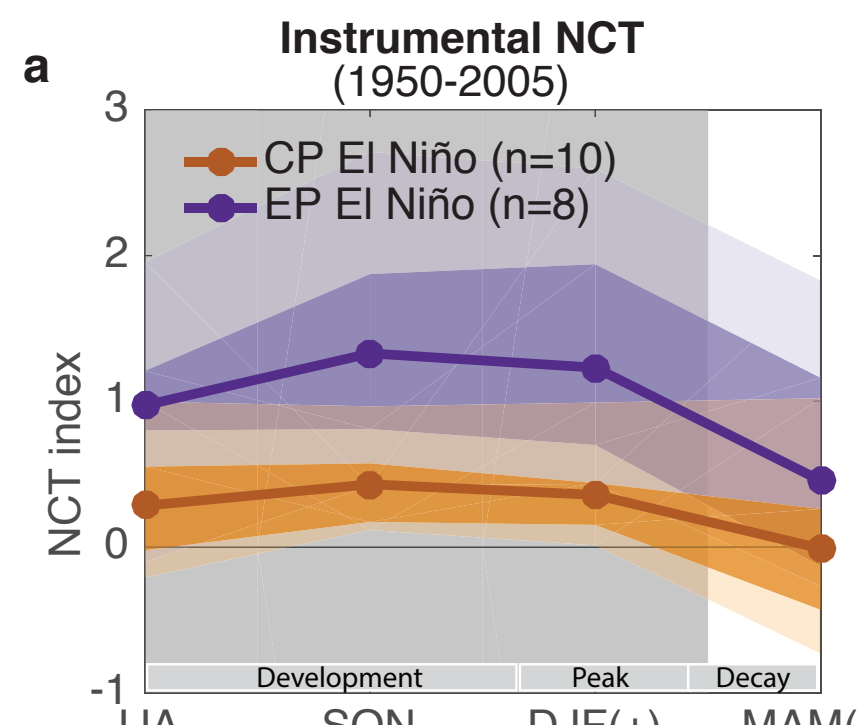

Coral network

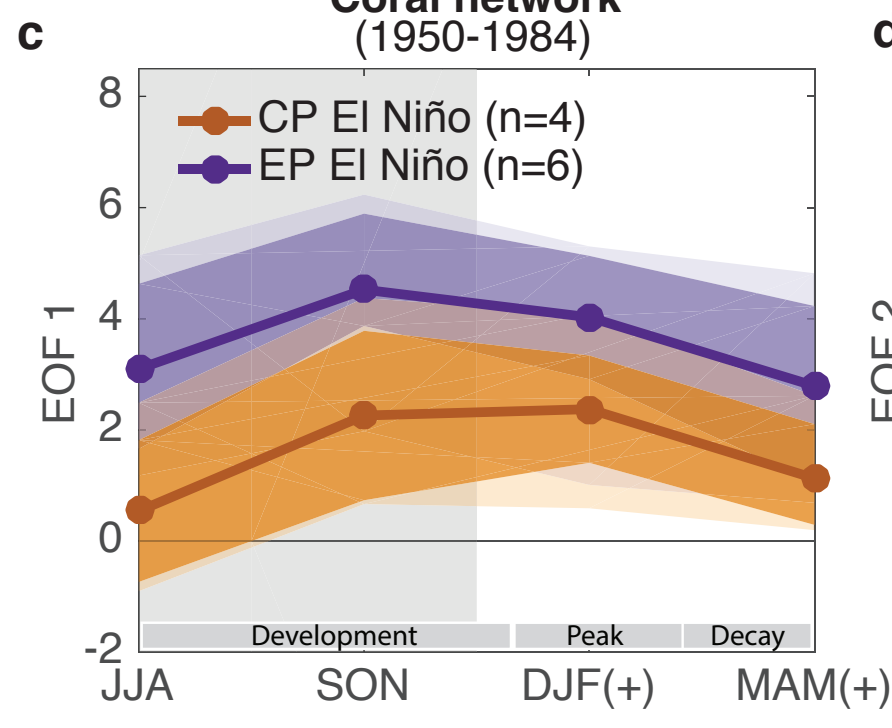

C

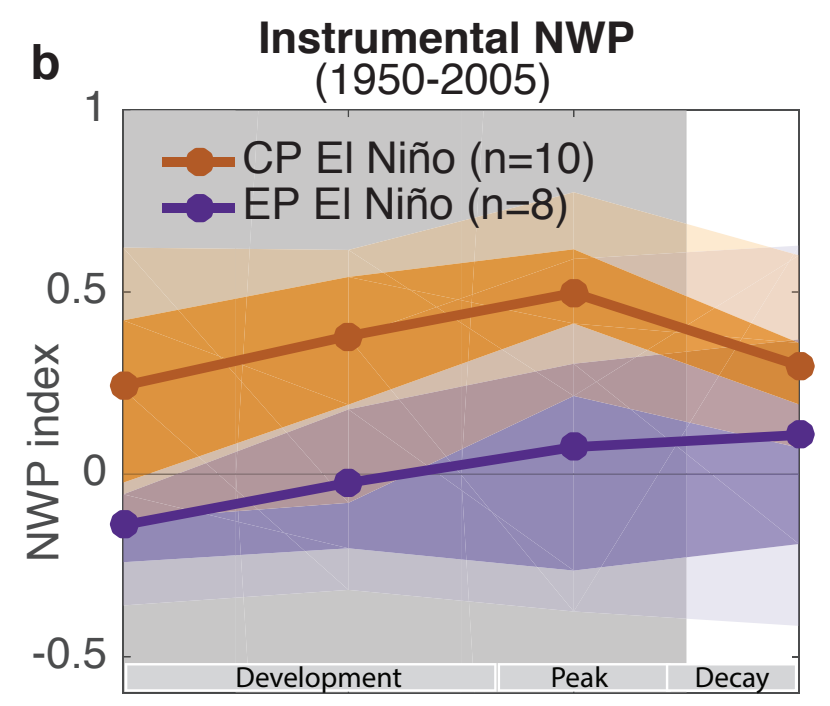

SON DJF(+)

Coral network

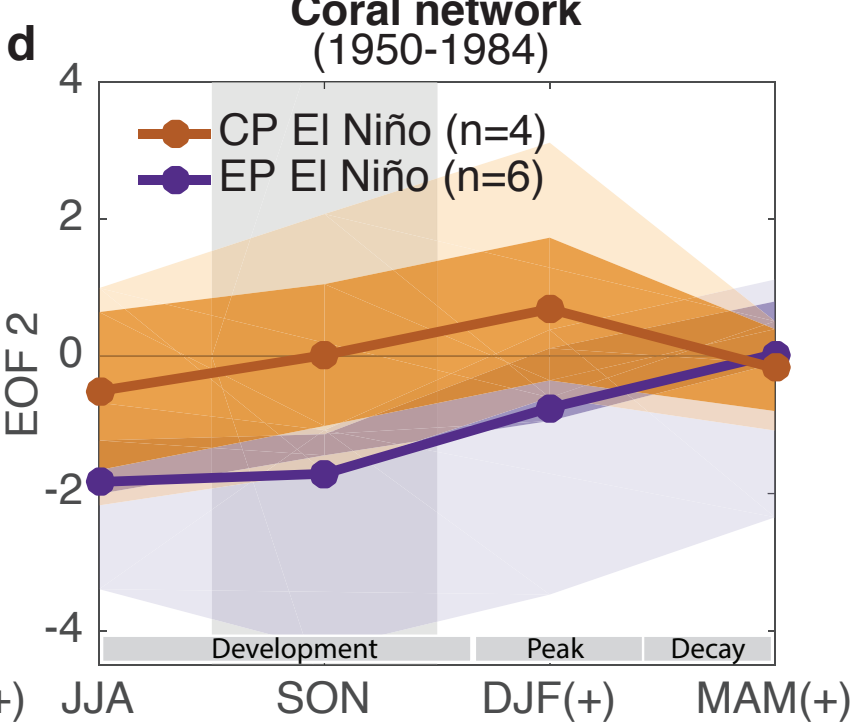

Figure 2: Seasonal distinction of EP and CP El Niño events. Instrumental composite of Niño Cold Tongue index (NCT) (a) and Niño Warm Pool index (NWP) (b) for EP and CP events (1950-2005) during different seasons. Coral network composite of the first (c) and second (d) EOF mode during EP and CP events (19501984). Average (line), $25^{\text {th }}-75^{\text {th }}$ percentile range (dark shading) and full range (light shading). Grey shading indicates seasons that are statistically different using a t-test (Instrumental:p $<0.05$, Coral network:p $<0.1$ ). 


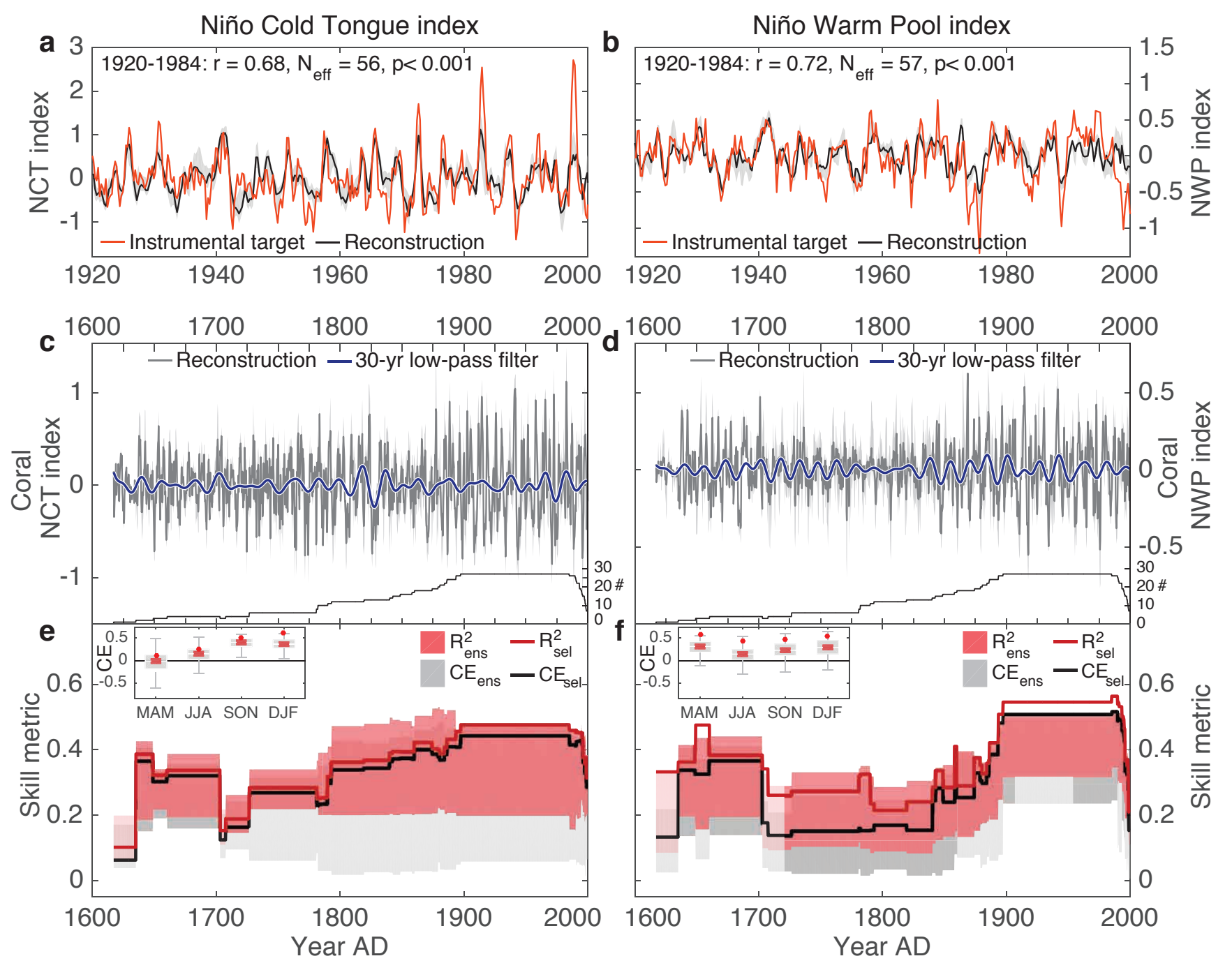

Figure 3: Reconstruction of seasonal El Niño indices. a, Comparison between the reconstructed (black line) and instrumental (orange line) Niño Cold tongue index (NCT) and $\mathbf{b}$, Niño Warm pool index (NWP) during instrumental period $\mathbf{c}, \mathbf{d}, \mathrm{NCT}$ and NWP seasonal reconstructions (dark lines), ensemble spread (light shading), 30-year low-pass filtered (blue lines) reconstructions and number of records. e,f, Verification statistics. Coefficient of Efficiency (CE) and explained variance $\left(R^{2}\right)$ for most replicated (sel, dark lines) and ensemble spread (ens, shaded envelopes). Insets show the median (red line), most replicated (red dot), interquartile range (grey boxes) and full range (whiskers) of ensemble spread of $\mathrm{CE}$ within seasons. 


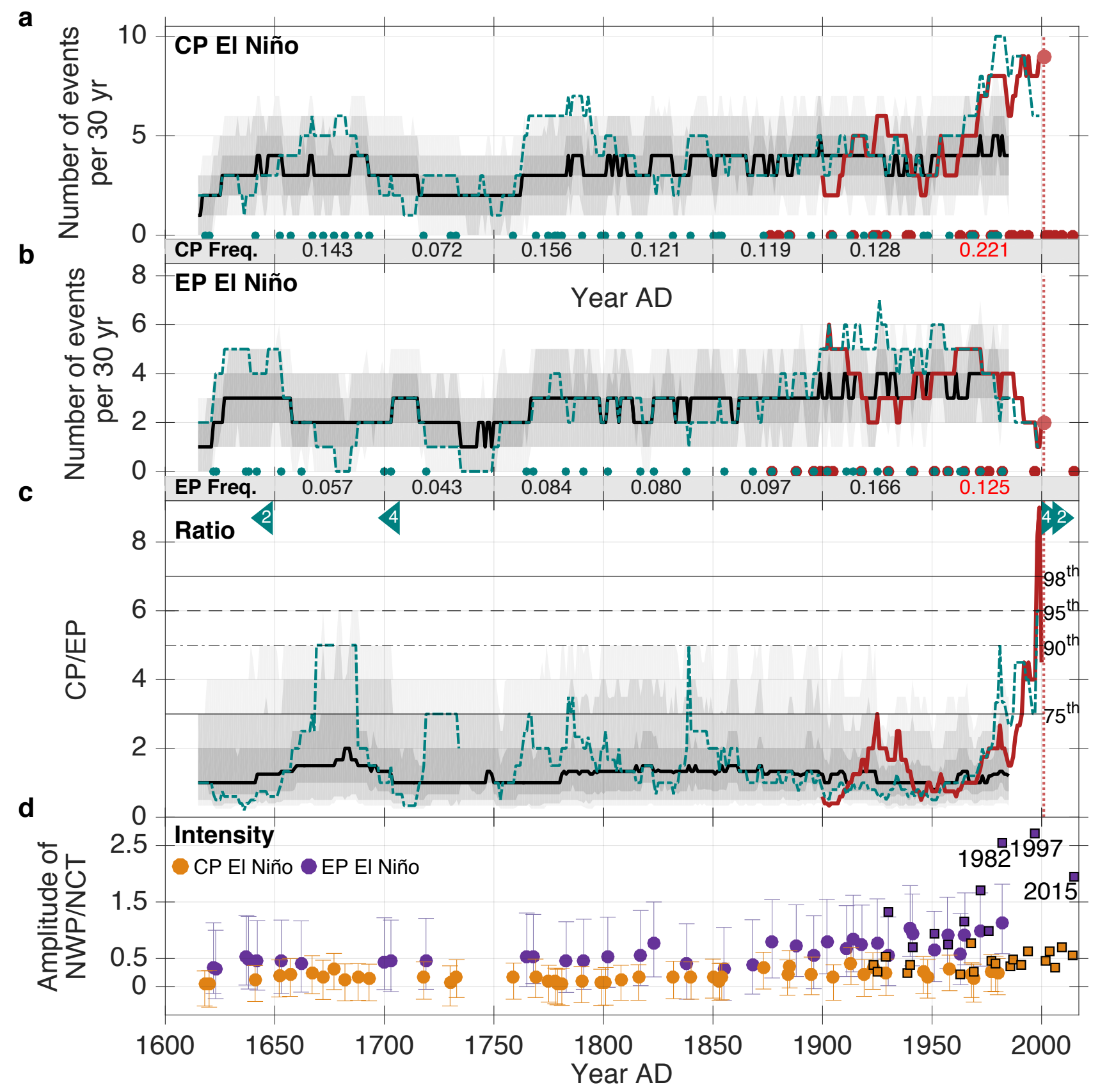

Figure 4: El Niño event diversity over the past four centuries. a,b,c, Number of CP El Niño (a), EP El Niño events (b) and ratio of CP to EP events (c) in sliding 30-yr windows; instrumental (red), mostreplicated reconstruction (teal), bootstrapped median (black) and uncertainty range in grey shadings $\left(75^{\text {th }}\right.$, $90^{\text {th }}, 95^{\text {th }}$ percentiles) shown. Event occurrence frequencies (per decade, insets) in 50-yr periods; individual events indicated by markers at zero line. Vertical red dotted line indicates most recent window, centred on 2001. d, Intensity of CP and EP El Niño events obtained by the NWP/NCT indices; instrumental events outlined in black; error bars indicate $95 \%$ confidence interval from bootstrapping. Small triangles show the number of contributing records when data availability is limited. 